\title{
Vos impôts sont-ils déjà optimalisés?
}

\section{6 questions sur la prévoyance professionnelle et le $3^{\mathrm{e}}$ pilier lié a}

Utilisez-vous déjà le montant maximum de votre $3^{\mathrm{e}}$ pilier lié a?

O OUI ONON

Montant maximum en 2008:

Vous êtes affilié à une caisse de pension (LPP): $\quad$ Fr. 6365.-

Vous n'êtes pas affilié à une caisse de pension (LPP): $20 \%$ du revenu AVS, max. Fr. 31 824.-

Avez-vous épuisé votre potentiel de rachat LPP?

Les rachats LPP peuvent être déduits entièrement de votre revenu imposable. Ainsi vous pouvez réaliser selon

le montant de votre revenu et selon le lieu d'imposition, une économie d'impôts jusqu'à $45 \%$ du rachat LPP.

Votre fortune est-elle bien placée fiscalement?

OUI O NON

Les gains de capitaux (p.ex. augmentation des cours) sont libres d'impôts, contrairement aux produits des capitaux (p.ex. intérêts) qui doivent être imposés comme revenu. Avec des solutions de prévoyances flexibles, les conseillers de FMH Insurance Services peuvent placer votre fortune globale, de telle manière que les produits des capitaux soient imposés par le fisc plus favorablement pour vous.

Avez-vous examiné s'il était possible d'augmenter la part épargne de votre LPP?

O OUI ONON

Lors d'une augmentation du montant de l'épargne de votre LPP, vous créez un nouveau potentiel de rachat qui vous permettra une optimalisation fiscale supplémentaire. Dans les solutions de prévoyance corporative le montant d'épargne peut s'élever jusqu'à $25 \%$ du salaire assuré.

Disposez-vous de plusieurs comptes ou assurances du $3^{\text {e }}$ pilier lié a?

O OUI ONON

Le versement des montants du $3^{e}$ pilier lié a est soumis à un impôt unique sur la prestation des capitaux. Le taux de cet impôt est souvent exponentiel. Si vous disposez par exemple de deux comptes de $3^{e}$ pilier lié a, vous pouvez retirer ces montants lors de deux périodes fiscales différentes et vous bénéficiez d'un taux moins élevé, d'où moins d'impôts à payer.

Votre époux/votre épouse dispose-t-il/elle également d'une LPP

O OUI O NON et d'un $3^{e}$ pilier lié a?

Souvent au cabinet le conjoint ne reçoit aucun salaire pour son activité et ainsi n'est pas affilié à une LPP. Si un salaire est versé au conjoint, celui-ci peut être affilié à une caisse de pension LPP et peut également conclure une prévoyance dans un $3^{\text {e }}$ pilier lié a.

\section{Evaluation}

$0 \times \mathrm{NON}$ : Sincères félicitations, vous avez déjà envisagé une optimalisation de votre prévoyance. Nous sommes à votre disposition pour voir si vous avez choisi les meilleures solutions.

1-6 $\times$ NON: Vous disposez d'un potentiel d'optimalisation important. Nous vous conseillons de vous adresser cette année encore à un spécialiste en prévoyance de FMH Insurance Services.

\section{Talon réponse}

\section{Prénom/Nom}

Adresse

NPA / Lieu

Date de naissance

Téléphone privé / cabinet

Atteignable le plus facilement (heure)

Adresse e-mail

Je désire un conseil personnalisé.

Prière de $m^{\prime}$ appeler.

Je m'intéresse à:
O $3^{\text {e }}$ pilier lié a
O Caisse de pension LPP
O Planification financière
O Responsabilité civile prof.
O Planification de la retraite $O$ Assurance protection juridique

O

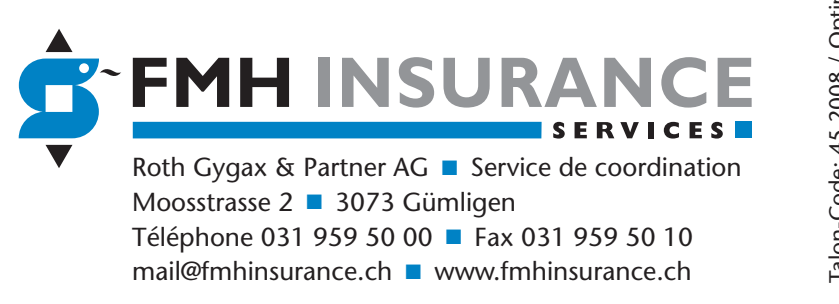

